\title{
KCa3.1 inhibition switches the phenotype of glioma-infiltrating microglia/macrophages
}

\author{
A Grimaldi', G D'Alessandro ${ }^{1,2}$, MT Golia ${ }^{1}$, EM Grössinger ${ }^{3}$, S Di Angelantonio ${ }^{1,4}$, D Ragozzino ${ }^{1,2}$, A Santoro ${ }^{5}$, V Esposito ${ }^{2,5}$, H Wulff ${ }^{3}$, \\ M Catalano ${ }^{1,2,7}$ and C Limatola ${ }^{2,6,7}$
}

Among the strategies adopted by glioma to successfully invade the brain parenchyma is turning the infiltrating microglia/ macrophages (M/MI) into allies, by shifting them toward an anti-inflammatory, pro-tumor phenotype. Both glioma and infiltrating M/MФ cells express the $\mathrm{Ca}^{2+}$-activated $\mathrm{K}^{+}$channel (KCa3.1), and the inhibition of $\mathrm{KCa} 3.1$ activity on glioma cells reduces tumor infiltration in the healthy brain parenchyma. We wondered whether $\mathrm{KCa} 3.1$ inhibition could prevent the acquisition of a pro-tumor phenotype by $\mathrm{M} / \mathrm{M} \Phi$ cells, thus contributing to reduce glioma development. With this aim, we studied microglia cultured in gliomaconditioned medium or treated with IL-4, as well as M/MФ cells acutely isolated from glioma-bearing mice and from human glioma biopsies. Under these different conditions, $\mathrm{M} / \mathrm{M} \Phi$ were always polarized toward an anti-inflammatory state, and preventing $\mathrm{KCa} 3.1$ activation by 1-[(2-Chlorophenyl)diphenylmethyl]-1H-pyrazole (TRAM-34), we observed a switch toward a pro-inflammatory, antitumor phenotype. We identified FAK and PI3K/AKT as the molecular mechanisms involved in this phenotype switch, activated in sequence after $\mathrm{KCa}$ 3.1. Anti-inflammatory $\mathrm{M} / \mathrm{M} \Phi$ have higher expression levels of $\mathrm{KCa} .1$ mRNA (kcnn4) that are reduced by $\mathrm{KCa} 3.1$ inhibition. In line with these findings, TRAM-34 treatment, in vivo, significantly reduced the size of tumors in glioma-bearing mice. Our data indicate that $\mathrm{KCa} 3.1$ channels are involved in the inhibitory effects exerted by the glioma microenvironment on infiltrating $\mathbf{M} / \mathbf{M \Phi}$, suggesting a possible role as therapeutic targets in glioma.

Cell Death and Disease (2016) 7, e2174; doi:10.1038/cddis.2016.73; published online 7 April 2016

Among primary brain tumors, glioblastoma multiforme (GBM) is the most aggressive form. Even after surgical ablation and routine treatments with chemo- and radio-therapeutic agents, patient survival remains $<15$ months. ${ }^{1}$ A wide spectrum of new therapeutic strategies is under investigation, including genetic and immunological approaches, but the need for new possible targets remains urgent. As in other tumors, the immune system has a central role in GBM, contributing to tumor progression and metastasis; ${ }^{2}$ for this reason, a novel research field is focused on immunotherapy-based drug discovery. Microglia represent important constituents of the innate immune system of the nervous system. However, in GBM, this first-line defensive system is hijacked by cytokines released by the tumor. ${ }^{3}$

Under physiological conditions, microglia continuously monitor the surrounding parenchyma with active movement of cell processes that extend and retract to sense potentially toxic local alterations. ${ }^{4,5}$ In addition to this patrolling phenotype, mainly associated with a ramified shape, microglia assume additional intermediate states, culminating in a fully amoeboid shape. ${ }^{6}$ The transition from ramified to amoeboid shape, together with the acquisition of specific functional and molecular characteristics, generates several intermediate phenotypes that represent different activation states: the extremes of this transition comprise, in analogy with the dichotomy described for macrophages, dynamic changes from a pro-inflammatory, M1-like phenotype, to an antiinflammatory one, namely $\mathrm{M} 2$, including a plethora of subphenotypes (M2a, M2b, M2c). ${ }^{7}$

Tumor-associated microglia/macrophages (M/MФ) are polarized toward an anti-inflammatory phenotype under the action of cytokines and factors released by tumor cells ${ }^{8-10}$ and contribute to further release of soluble factors, which influence tumor growth and behavior. ${ }^{11,12} \mathrm{~A}$ wide range of studies aim to induce tumor- associated macrophages to switch from the pro-tumor to the antitumor pro-inflammatory phenotype. ${ }^{13}$

One promising target to control microglia phenotype is the $\mathrm{Ca}^{2+}$-activated $\mathrm{K}^{+}$channel (KCa3.1), which is involved in microglia activation in different pathological conditions such as glioma, ${ }^{14}$ spinal cord injury, ${ }^{15}$ ischemia ${ }^{16}$ and Alzheimer's Disease. ${ }^{17}$ In particular, KCa3.1 blockade reduced infarct area and glioma invasiveness in rodent models of cerebral

\footnotetext{
${ }^{1}$ Department of Physiology and Pharmacology, Sapienza University of Rome, Piazzale Aldo Moro 5, Rome 00185, Italy; ${ }^{2}$ IRCCS Neuromed, Via Atinense 18, Pozzilli 86077, Italy; ${ }^{3}$ Department of Pharmacology, University of California, 451 Health Sciences Drive, GBSF3502, Davis, CA 95616, USA; ${ }^{4}$ Center for Life Nanoscience Istituto Italiano di Tecnologia@Sapienza, Rome, Italy; ${ }^{5}$ Department of Neurology and Psychiatry, Sapienza University of Rome, Piazzale Aldo Moro 5, Rome 00185, Italy and ${ }^{6}$ Pasteur Institute-Department of Physiology and Pharmacology, Sapienza University of Rome, Piazzale Aldo Moro 5, Rome 00185, Italy

*Corresponding author: C Limatola, Department of Physiology and Pharmacology, Sapienza University of Rome, Piazzale Aldo Moro, 5; Rome 00185, Italy; Tel: +39 06 4969 0243; Fax: +39 064991 0851; E-mail: cristina.limatola@uniroma1.it

${ }^{7}$ These authors contributed equally to this work.

Abbreviations: $\mathrm{M} / \mathrm{M} \Phi$, microglia/macrophages; KCa3.1, $\mathrm{Ca}^{2+}$ activated $\mathrm{K}^{+}$channel; GBM, glioblastoma multiforme; TRAM-34, 1-[(2-Chlorophenyl)diphenylmethyl]-1Hpyrazole; IL, interleukin; LPS, lipopolysaccharide; IFN, interferon; FAK, focal adhesion kinase; PI3K, phosphoinositide-3 kinase; Akt, protein kinase B; GCM, gliomaconditioned medium; NCM, non-conditioned medium; RT-PCR, real-time PCR; PAP-1, 5-(4-phenoxybutoxy)psoralen; Kv1.3, voltage-activated K ${ }^{+}$channel; SKA-31, naphtho [1,2-d]thiazol-2-ylamine

Received 13.11.15; revised 22.2.16; accepted 02.3.16; Edited by G Melino
} 
ischemia and cerebral tumor ${ }^{14,16}$ and reduced the neurotoxicity of microglia upon lipopolysaccharide (LPS) or oligomeric amyloid $\beta$ exposure. ${ }^{18,19}$ Interestingly, KCa3.1 expression level negatively correlates with patient prognosis. ${ }^{20}$

In this paper, we investigated the effect of $\mathrm{KCa} 3.1$ inhibition on the phenotype switching of microglia induced by GBMconditioned medium (GCM) or by interleukin (IL)-4, a cytokine involved in microglia polarization toward the anti-inflammatory phenotype. We demonstrated that, upon $\mathrm{KCa} 3.1$ inhibition by 1-[(2-Chlorophenyl)diphenylmethyl]-1H-pyrazole (TRAM-34), the GBM- or IL-4-induced profile of $\mathrm{M} / \mathrm{M} \Phi$ is more polarized toward an inflammatory phenotype. This effect is confirmed, ex vivo, in human specimens from glioma patients and, in vivo, in a mouse model of glioma, where TRAM-34 treatment induced an increase of pro-inflammatory and a reduction of anti-inflammatory gene expression in infiltrating CD11 $\mathrm{b}^{+}$cells. This polarization corresponded to a reduced tumor size in TRAM-34-treated glioma-bearing mice, further supporting the antitumor effect of inflammatory $M / M \Phi$ cells. We also described the molecular mechanisms involved in these modulatory effects, demonstrating the involvement of the focal adhesion kinase (FAK) and phosphoinositide-3 kinase/ protein kinase $\mathrm{B}$ (PI3K/AKT) signaling pathways.

\section{Results}

We have previously demonstrated that KCa3.1 expression on glioma cells is involved in tumor cell migration in cerebral parenchyma. ${ }^{14}$ As KCa3.1 channels in the brain are also expressed by microglia and infiltrating macrophages $(M / M \Phi)$, we now investigated the effect of KCa3.1 blockage on $M / M \Phi$ cells, with particular interest on the acquisition of pro-tumor or antitumor phenotype.

It is known that the $\mathrm{M} / \mathrm{M} \Phi$ cell population is deeply influenced by soluble factors produced by tumor cells. ${ }^{9,10}$ Data in Figure 1a indicate that CD11b+ cells (mainly represented by $\mathrm{M} / \mathrm{M} \Phi$ ) isolated from the brain hemisphere ipsilateral to glioma cells injection do express typical antiinflammatory genes (arg1, ym1, fizz1, cd163 and cd206), with pro-tumor activity that, upon TRAM-34 treatment, undergo a significant reduction of expression. Interestingly, pro-inflammatory, anti-tumor genes (cd86, tnfa, inos, il1 $\beta$ and il6), whose expression is not different in the two cerebral hemispheres, regardless of tumor presence (with the only exception being represented by inos), are remarkably increased in the glioma ipsilateral hemisphere of TRAM-34-treated mice. Interestingly, when TRAM-34-treated mice were analyzed for tumor growth, a significant reduction of tumor volume was observed (Figure 1b).

Similar results were obtained when CD11b+ cells were isolated from tumoral tissue obtained from GBM patients undergoing surgery: upon TRAM-34 treatment, these cells had a significant reduction of human anti-inflammatory markers (CD163, MMP12) and a significant increase of human pro-inflammatory markers (CXCL10, IL12A, NOS2 and TNF) ${ }^{21}$ (Figure 1c), confirming in human GBM the data obtained in glioma-bearing mice.

To better investigate the molecular mechanisms involved in this potassium channel-dependent phenotype switch of $\mathrm{M} / \mathrm{M} \Phi$, we used primary murine microglia exposed to $\mathrm{GCM}$ or non-conditioned medium (NCM) for $24 \mathrm{~h}$. Data shown in Figure 2 a show that, similarly to what observed ex vivo, cell exposure to GCM induced an increased expression of anti-inflammatory genes; TRAM-34 treatment reduced their expression and increased the transcription of proinflammatory genes. Similar phenotypic switch was observed when cultured microglia were polarized by cytokine treatment (48 $\mathrm{h}$ with LPS/interferon- $\gamma$ (IFN $\gamma$ ) or IL-4) and treated with TRAM-34; also, in these conditions the expression of anti-inflammatory genes was reduced with an increase of proinflammatory genes upon KCa3.1 inhibition (Supplementary Figure S1A).

The activation state of microglia has been often correlated with their shape, although it is not possible to strictly associate a morphology to a specific phenotype. ${ }^{6}$ A ramified microglia has often been related to a less active, resting phenotype, in comparison with round, amoeboid, active cells. However, this simplified view has been challenged by the description of the continuous monitoring activity of ramified microglia, demonstrating that these cells are not really resting and should probably rather be called 'surveillant'. ${ }^{4}$ We measured the ramification grade of microglia calculating the 'form factor', a parameter taken as 1 for round cells and correspondingly $<1$ when the morphology deviates from the spherical shape. Figure $2 \mathrm{~b}$ shows that microglia exposed to GCM have a decreased form factor versus NCM-exposed cells and that TRAM-34 treatment significantly reverts this effect. Similar results were obtained when microglia were treated in vitro with LPS/IFN $\gamma$ or IL-4 (Supplementary Figure S1B).

To test whether microglia phenotypes correlate with different expression levels of KCa3.1, real-time PCR (RT-PCR) analyses were performed on $C D 11 b^{+}$cells isolated from the brain of GL261-bearing mice, or from human GBM specimens, and on NCM- and GCM-exposed or cytokine-polarized microglia. A significant higher expression of KCa3.1 (kcnn4 gene) was observed in $\mathrm{CD}_{11 \mathrm{~b}^{+}}$cells isolated from the ipsilateral cerebral hemisphere of glioma-bearing mice in comparison with the contralateral hemisphere and this difference was totally abolished by TRAM-34 treatment (Figure 3a, left). The expression level of kcnn4 in CD11b+ cells isolated from the contralateral hemisphere was always comparable with the level measured in control healthy mice (data not shown). In accordance, KCa3.1 level was reduced upon TRAM-34 treatment in M/M $\Phi$ cells (lba1+) infiltrating the tumor area (Figure $3 \mathrm{a}$, center and right). Consistently, when $\mathrm{CD}_{11 \mathrm{~b}^{+}}$cells isolated from human GBM specimens were treated with TRAM-34 for $24 \mathrm{~h}$, a significant reduction of KCNN4 expression was observed (Figure $3 b$ ). Similar results were obtained on cultured microglia upon GCM (Figure 3c) and IL-4 (Figure 3d) treatment, and KCa3.1 inhibition abolished the effect. Taken together, these data suggest that the activity of $\mathrm{KCa} 3.1$ channels modulates both microglia phenotype and channel expression.

Modulation of channel expression was confirmed by functional analysis: cytokine-polarized microglia were tested for KCa3.1-mediated currents by patch-clamp recordings. Data shown in Figure $3 e$ demonstrate the presence of large, 5-(4-phenoxybutoxy)psoralen (PAP-1)-sensitive voltageactivated $\mathrm{K}^{+}$channel (Kv1.3) currents for LPS/IFN $\gamma$-treated microglia, together with barely detectable TRAM-34-sensitive 

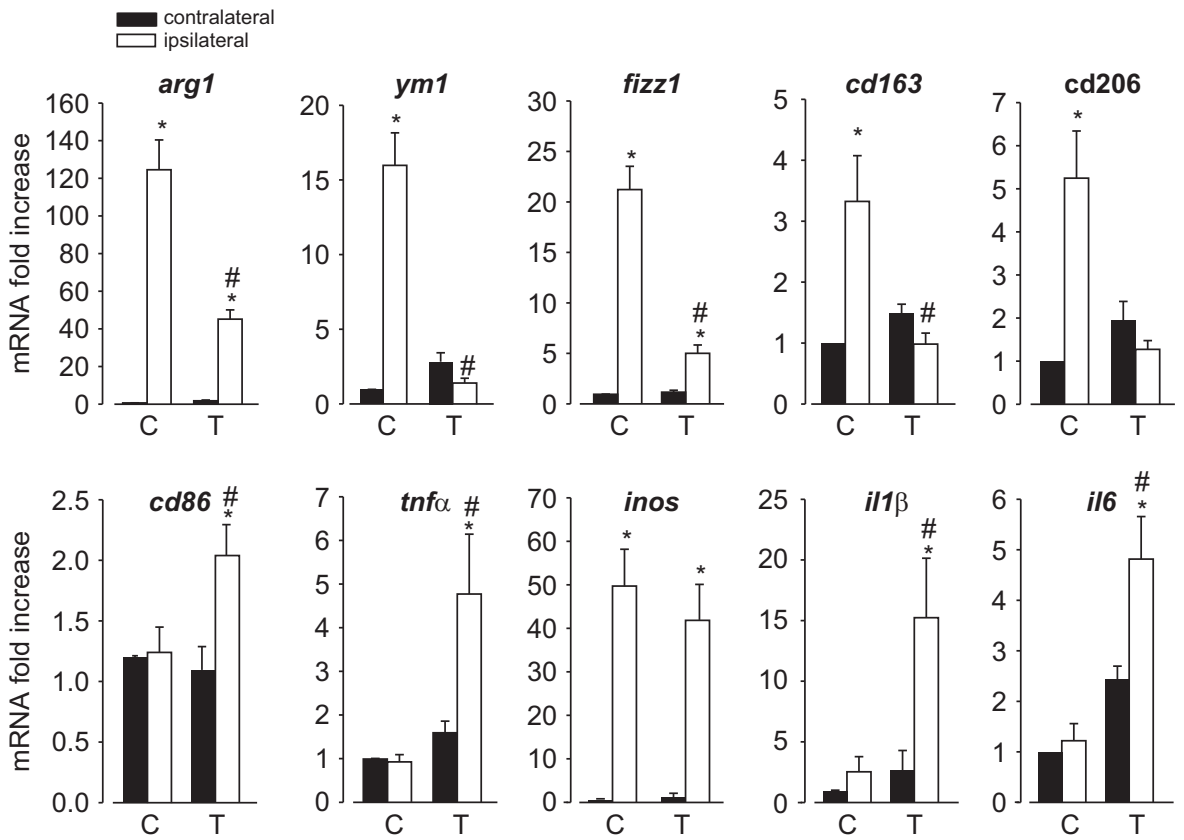

b
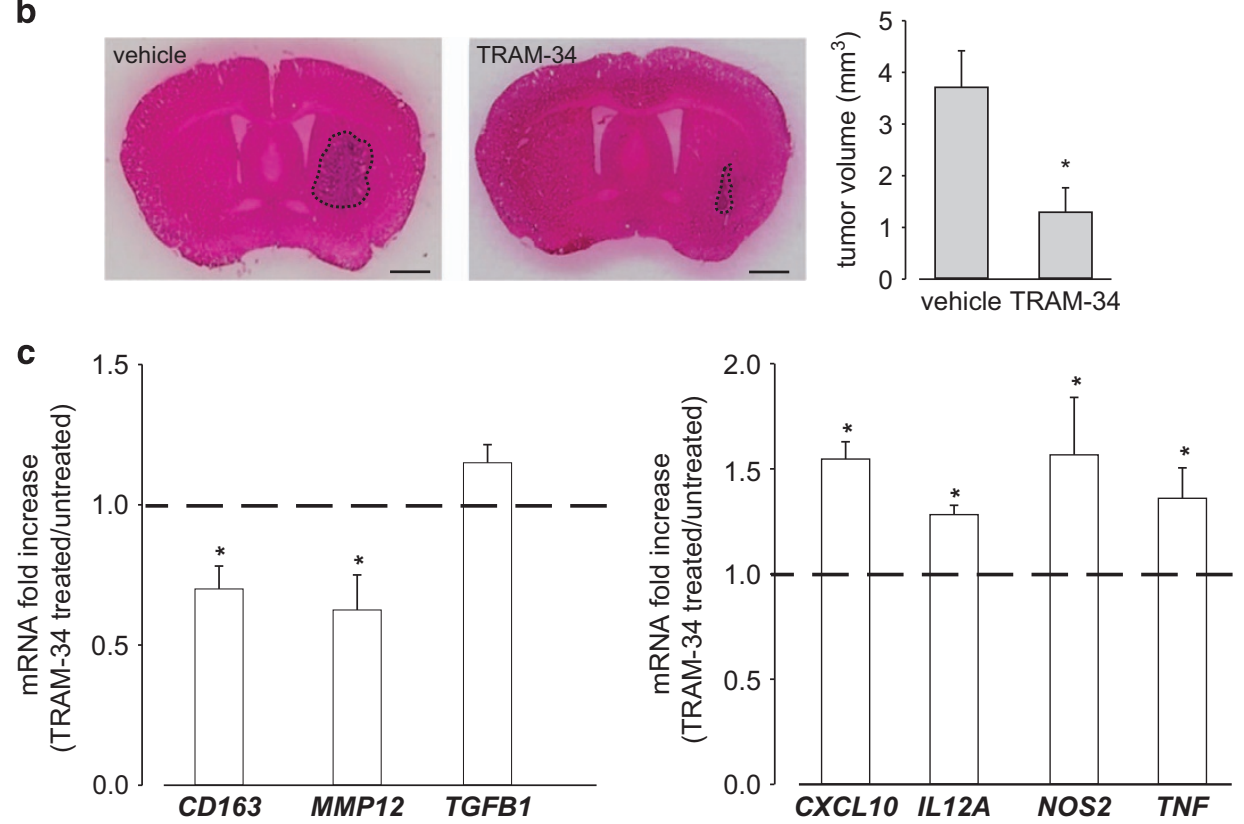

Figure $1 \mathrm{KCa3.1}$ inhibition modifies the polarization of CD11 $\mathrm{b}^{+}$cells extracted from glioma-bearing mice and from biopsies of human GBM. (a) RT-PCR of M2-like (arg1, ym1, fizz1, cd163 and cd206) and M1-like (cd86, tnfo, inos, il1 $\beta$ and il6) related genes in CD11 b ${ }^{+}$cells extracted from ipsilateral and contralateral cerebral hemispheres of GL261bearing mice treated with vehicle (C) or TRAM-34 (T, $120 \mathrm{mg} / \mathrm{kg} / \mathrm{die}$ ). Data are the mean \pm S.E.M., ${ }^{*} P<0.05$ versus $\mathrm{C}$ contralateral; ${ }^{\#} P<0.05$ versus $\mathrm{C}$ ipsilateral by two-way ANOVA; $N=8$. (b) Tumor volumes in the brain of mice treated with vehicle or TRAM-34. Data are the mean + S.E.M., ${ }^{*} P<0.05$ by Student's t-test; $N=8$. Scale bar: $1 \mathrm{~mm}$. (c) RT-PCR for human M2-like (CD163, MMP12, TGFB1) and M1-like (CXCL10, IL12A, NOS2, TNF) related genes expressed by CD11b+ cells extracted from human GBM specimens and treated for $24 \mathrm{~h}$ with TRAM-34 $(2.5 \mu \mathrm{M})$. Data are expressed as fold change of TRAM-34-treated versus untreated samples and are the mean \pm S.E.M., ${ }^{\star} P<0.05$ by Student's t-test; $N=4$

currents (inset, $16.05 \pm 7.34 \mathrm{pS} / \mathrm{pF}$ ). In contrast, IL-4-treated microglia had no significant Kv1.3 currents (not shown), with a more prominent TRAM-34-sensitive component $(19.45 \pm 12.68 \mathrm{pS} / \mathrm{pF})$ confirming the tendency to a preferential expression of these channels on microglia with antiinflammatory features.
We have previously shown that GCM exposure modulates microglia migration and phagocytic activity and that KCa3.1 inhibition abolished these effects. ${ }^{14}$ We confirmed these data by performing migration, invasion and phagocytosis assays on primary murine microglia exposed to medium conditioned by GL261 (Figure 4a), U87MG and GL15 (Supplementary 

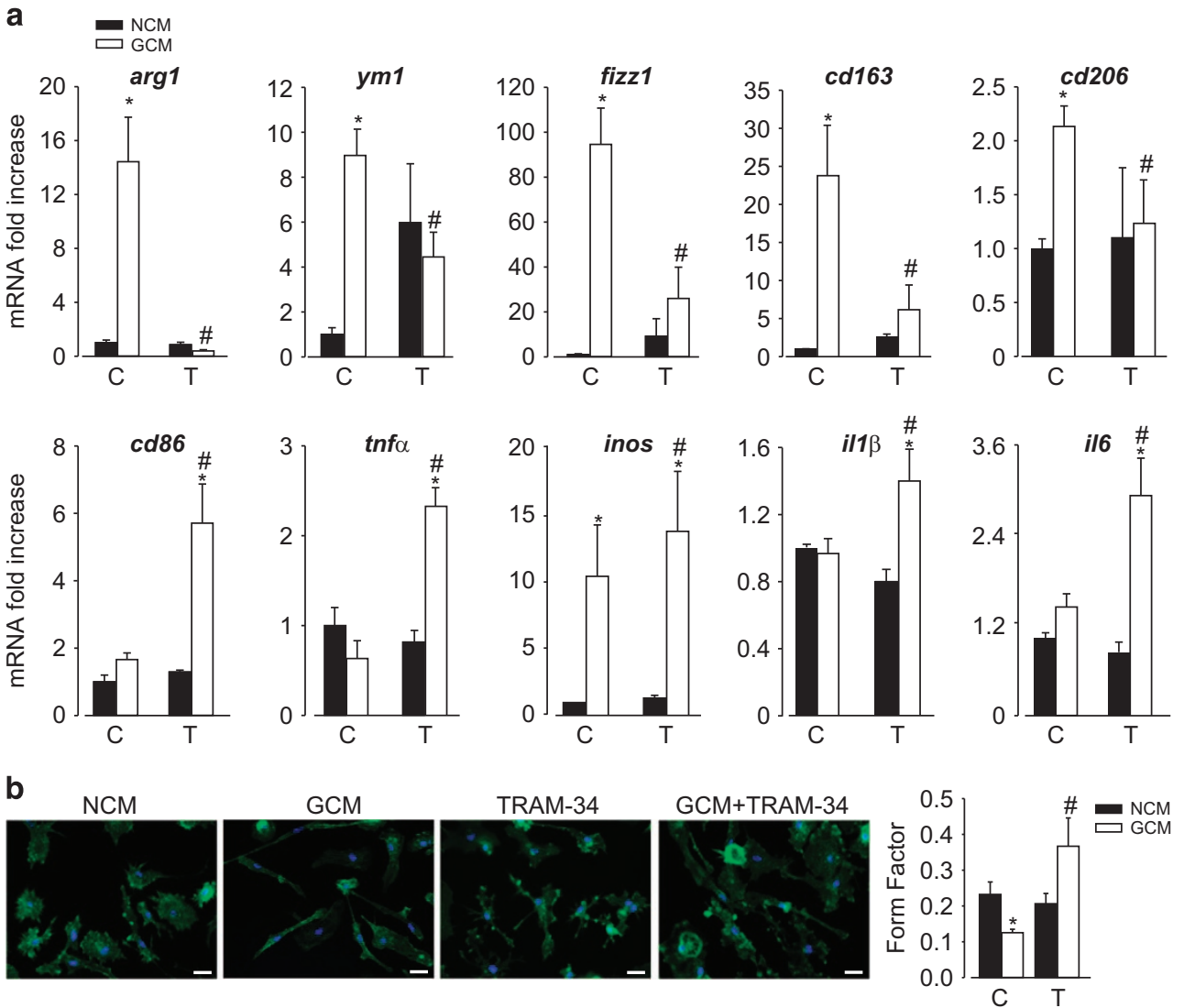

Figure 2 KCa3.1 inhibition reverts GCM-induced microglia polarization. (a) RT-PCR on mRNAs of microglia treated with NCM or GCM in the absence (C) or presence of TRAM-34 (T, $2.5 \mu \mathrm{M})$, analyzed for the expression of M2-like (arg1, ym1, fizz1, cd163 and cd206) and M1-like (cd86, tnfo, inos, il1 $\beta$ and il6) related genes. Data are expressed as fold increase and are the mean \pm S.E.M.; ${ }^{*} P<0.05$ versus NCM; ${ }^{\#} P<0.05$ versus GCM/C by two-way ANOVA and paired $t$-test for inos; $N=8$. (b) NCM- and GCM-treated microglia in the absence (C) or presence of TRAM-34 (T, $2.5 \mu \mathrm{M})$, stained with phalloidin (green) and Hoechst (blue). Scale bar: $20 \mu \mathrm{m}$. Form factor values (calculated as reported in the Materials and Methods section) are shown in the graph and are the mean \pm S.E.M., ${ }^{*} P<0.05$ versus NCM; ${ }^{\#} P<0.05$ versus GCM/C by two-way ANOVA; $N=4$

Figure S2) glioma cell lines, in the presence of TRAM-34, finding that $\mathrm{KCa} 3.1$ block inhibited all the activities potentiated by GCM (Figure 4a). To further support these results, we used a specific activator of $\mathrm{KCa} 3.1$, naphtho[1,2-d]thiazol-2-ylamine (SKA-31), and found that treatment with this compound increased microglia migration, invasion and phagocytic activity (Figure 4b). Moreover, we analyzed the same activities in primary murine microglia treated with LPS/IFN $\gamma$ or IL-4, demonstrating that only IL-4-treated microglia had increased phagocytic, chemotactic and invasive properties (similarly to GCM-exposed microglia). KCa3.1 inhibition impaired these functional modulations, being ineffective on LPS/IFN $\gamma$-stimulated microglia (Figure 4c). To further investigate this effect, we decided to study whether cytokine stimulation modulated the expression levels of receptors for chemokines or MMPs. IL-4 treatment induced increased expression of CXCR4, CXCR6 and MMP-9 on microglia, while LPS/IFNy treatment was ineffective (on CXCR6 and MMP-9) or drastically inhibitory (on CXCR4). TRAM-34 abolished the effects of IL-4 on chemokine receptors and MMP-9 expression (Supplementary Figure S3A). Consistently, IL-4-treated cells migrated more toward CXCL12 and CXCL16 (Supplementary Figure S3B). MTT assay excluded "that the effects of GCM and IL-4 was on cell proliferation," some increase being only observed for LPS/ IFN $\gamma$-treated cells (Supplementary Figure S3C).

It has been demonstrated that the exposure of microglia to soluble factors released by glioma cells induces the activation of the PI3K/Akt and FAK pathways and that these signaling pathways are involved in cell movement and phagocytosis. ${ }^{22,23}$ Having demonstrated that anti-inflammatory microglia have increased migratory and phagocytic activity (Figure 4c) and higher functional expression of kcnn4 (Figure 3), we wanted to verify the hypothesis that $\mathrm{KCa3.1}$ activity could contribute to the phenotype switch of microglia through the modulation of these signaling pathways. We observed that GCM-exposed microglia have increased FAK and AKT phosphorylation, significantly reduced by TRAM-34 treatment, demonstrated both by western blotting and immunofluorescence analysis (Figure 5a and Supplementary Figure S4). Moreover, GCM-induced migration, invasion and phagocytosis were completely inhibited in the presence of FAK (PF-228) and PI3K/AKT (LY294002) inhibitors (Figure 5b). To investigate whether KCa3.1 activation has effect on FAK and PI3K/AKT pathways, cultured microglia were treated with SKA-31, a channel activator. Data shown in Figure $5 \mathrm{c}$ demonstrate that both FAK and AKT 
a

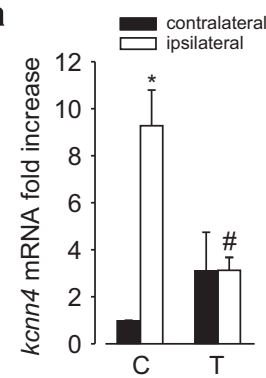

b

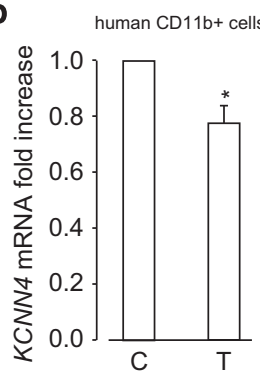

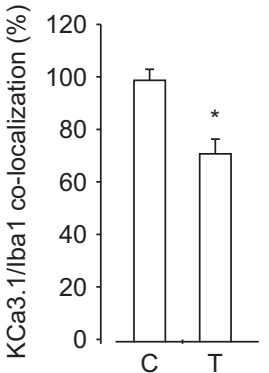

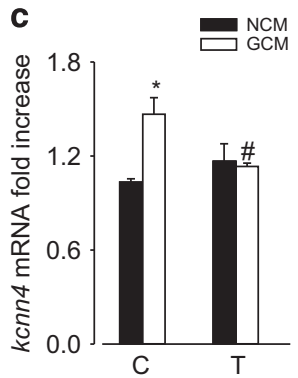

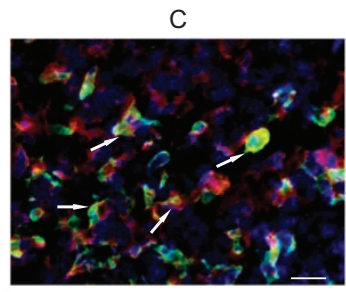

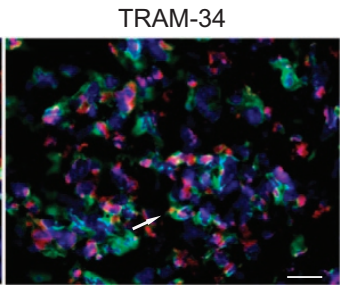

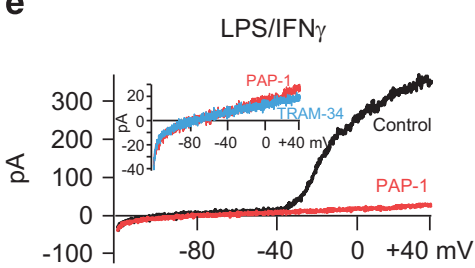

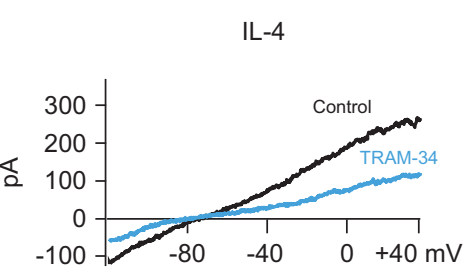

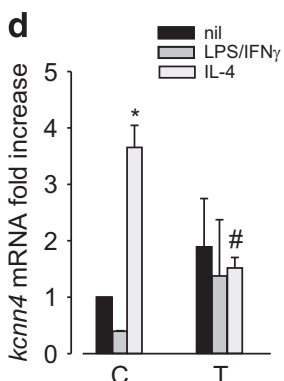

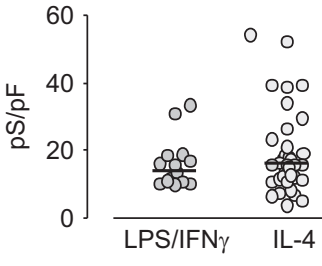

Figure $3 k c n n 4$ expression in microglia and infiltrating M/M $\Phi$ is correlated with different activation states. (a, left) RT-PCR of $k c n n 4$ expression in CD11b+ cells extracted from the ipsilateral and contralateral cerebral hemispheres of glioma-bearing mice treated with vehicle (C) or TRAM-34 (T, $120 \mathrm{mg} / \mathrm{Kg} / \mathrm{die})$. Data are the mean $\pm \mathrm{S} . \mathrm{E} . \mathrm{M}$., ${ }^{*} P<0.05$ versus ipsilateral, ${ }^{\#} P<0.05$ versus contralateral/C by two-way ANOVA; $N=8$. (a, center) KCa3.1 expression in Iba1 ${ }^{+}$cells in the tumor area of glioma-bearing mice treated with vehicle $(C)$ or TRAM-34 (T, $120 \mathrm{mg} / \mathrm{Kg} / \mathrm{die})$. Data are the average fluorescence intensity of merged signals \pm S.E.M. and are expressed as the percentage of $\mathrm{C},{ }^{*} P<0.05$ by Student's $t$-test; $N=4$. Representative images are shown on the right (KCa3.1 in red, Iba1 in green), with the arrows indicating some merged signals; scale bar $=20 \mu \mathrm{m}$. (b) RT-PCR of KCNN4 expression in CD11b cells extracted from human GBM specimens untreated (C) or treated with TRAM-34 (T, $2.5 \mu \mathrm{M}$ ) for $24 \mathrm{~h}$. Data are expressed as fold change of TRAM-34-treated versus untreated samples and are the mean \pm S.E.M. ${ }^{*} P<0.05$ by Student's $t$-test; $N=4$. (c) RT-PCR of $k c n n 4$ expression in microglia exposed to NCM and GCM or (d) treated with LPS/IFN $\gamma$ and IL-4 in the absence (C) or presence of TRAM-34 (T, $2.5 \mu \mathrm{M})$; ${ }^{*} P<0.05$ versus NCM, $\# P<0.05$ versus GCM/C by two-way ANOVA in (c); ${ }^{*} P<0.05$ versus nil, $\# P<0.05$ versus LPS/IFN $\gamma / C$ or versus IL4/C by two-way ANOVA in (d); data are expressed as fold increase and are the mean \pm S.E.M.; $N=4$. (e) Typical current traces in response to repeated voltage ramps from -120 to $+40 \mathrm{mV}$ (holding potential $-80 \mathrm{mV}$ ). Each panel shows superimposed currents from microglia treated with LPS/IFN $\gamma$ and IL-4 before (black) and after TRAM-34 (1 $\mu \mathrm{M}$, blue) perfusion. The prominent Kv current in LPS/IFN $\gamma$ treated microglia was first blocked with $1 \mu \mathrm{M}$ PAP-1. On the right, scattered plot representing TRAM-34-sensitive current density in LPS/IFN $\gamma(N=15)$ and IL-4 ( $N=35)$ treated microglia

phosphorylation was higher in SKA-31-treated cells. As FAK activation has been demonstrated to be upstream to the PI3K/ AKT pathway in macrophages, ${ }^{24,25}$ we investigated the crosstalk among these kinases in microglia upon KCa3.1 channel activation. In the presence of PF-228, the effect of SKA-31 on FAK and AKT phosphorylation was inhibited while, in the presence of LY294002, the SKA-31-dependent FAK phosphorylation was maintained, being AKT phosphorylation inhibited (Figure 5c). These data indicate that KCa3.1 activation is upstream to FAK activation and that FAK precedes AKT phosphorylation.

To define the role of the FAK/PI3K/AKT pathway in phenotype polarization, microglia were treated with NCM or GCM and LPS/IFN $\gamma$ or IL-4 and incubated with PF-228 or LY294002. Both kinase inhibitors were ineffective on NCM- and LPS/IFN $\gamma$-treated microglia but prevented GCM- and IL-4-induced expression of anti-inflammatory genes (Figure 6).

\section{Discussion}

GBM is the brain tumor with the lowest survival rate from the time of diagnosis. Its aggressiveness is certainly aided by the ability to produce cytokines and factors involved in tuning down the local immune system response, resulting in an inability to react against cancer cell proliferation and invasion. ${ }^{26}$ One approach to fight GBM is to re-activate the immune system starting with local microglia and infiltrating macrophages, which constitute the first-line innate immune defense in the brain.

In this study, we tested the hypothesis that targeting microglial KCa3.1 channels could represent a therapeutic 

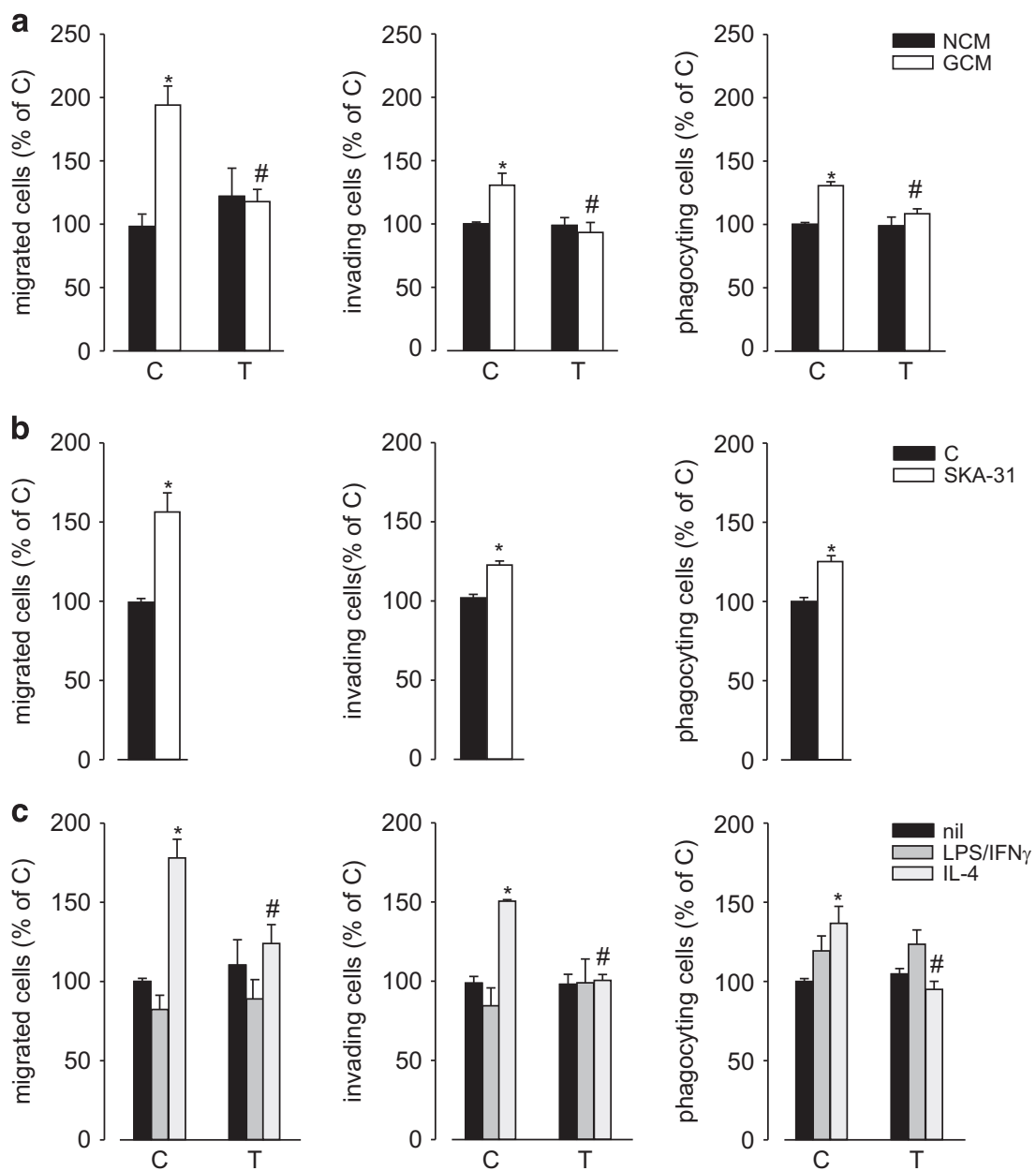

Figure $4 \mathrm{KCa} .1$ modulates microglia functional activities. (a) NCM- and GCM-treated microglia, in the absence (C) or presence of TRAM-34(T, 2.5 $\mu \mathrm{M})$ assayed for migration, invasion and phagocytosis. Data are the mean \pm S.E.M., ${ }^{*} P<0.05$ versus NCM; ${ }^{\#} P<0.05$ versus GCM/C by two-way ANOVA; $N=4$. (b) Untreated (C) or SKA-31 $(250 \mathrm{nM})$ treated microglia assayed for migration, invasion and phagocytosis. Data are the mean \pm S.E.M., ${ }^{*} P<0.05$ by Student's $t$-test; $N=4$. (c) Untreated, LPS/IFN $\gamma$ - or IL-4treated microglia in the absence $(\mathrm{C})$ or presence of TRAM-34 (T, $2.5 \mu \mathrm{M})$ assayed for migration, invasion and phagocytosis. Data are the mean \pm S.E.M., ${ }^{*} P<0.05$ versus nil; ${ }^{\#} P<0.05$ versus $\mathrm{LPS} / \mathrm{IFN} \gamma / \mathrm{C}$ or IL4/C by two-way ANOVA; $N=4$

strategy to counteract GBM malignancy, helping microglia to re-acquire a more pro-inflammatory, antitumor phenotype. This hypothesis is supported by several recent findings demonstrating the ability of the tumor microenvironment to hamper microglia inflammatory reaction, inhibiting the maintenance of the M1-like (pro-inflammatory) phenotype initially induced upon microglia interaction with glioma cells. We demonstrated that the anti-inflammatory $M / M \Phi$ phenotype induced by interaction with glioma could be significantly attenuated, in favor of a pro-inflammatory state by modulating the activity of KCa3.1.

Microglia cells isolated from glioma-bearing mice treated with TRAM-34 had a phenotype clearly oriented toward the pro-inflammatory, antitumor state, consistent with the reduction of tumor size observed in these animals. We cannot exclude that, at least in vivo, the TRAM-34-induced phenotype switch of $M / M \Phi$ could be secondary to a direct effect of the drug on glioma. ${ }^{14}$ However, this would only partially explain the effect, because similar effects on cell phenotype were obtained with CD11b+ cells from human GBM biopsies treated with TRAM-34 and with pure cultured microglia exposed to GCM or IL-4. These data indicate that the block of KCa3.1 is sufficient to hinder microglia phenotype switch, suggesting that these channels could be directly involved in modulating the microglia activation state. This is in line with previous papers demonstrating that $\mathrm{KCa} 3.1$ inhibition affects microglia proliferation, p38 MAPK phosphorylation, NF- $k$ B activation and nitric oxide generation. ${ }^{18,19}$ Similar results were obtained when microglia were stimulated with cytokines: microglia treated with IL-4 displayed increased levels of the antiinflammatory genes arg1, ym1, fizz1, cd163 and cd206, which were all reduced upon TRAM-34 treatment. Consistently, in IL-4-treated cells, we observed higher levels of kcnn4 and recorded TRAM-34-sensitive currents.

Taken together, these data support the hypothesis that $\mathrm{KCa} 3.1$ is involved in determining microglia phenotype, in particular shifting glioma-induced cell polarization toward a pro-inflammatory state. Interestingly, a shift of IL-4-polarized 


\section{a}
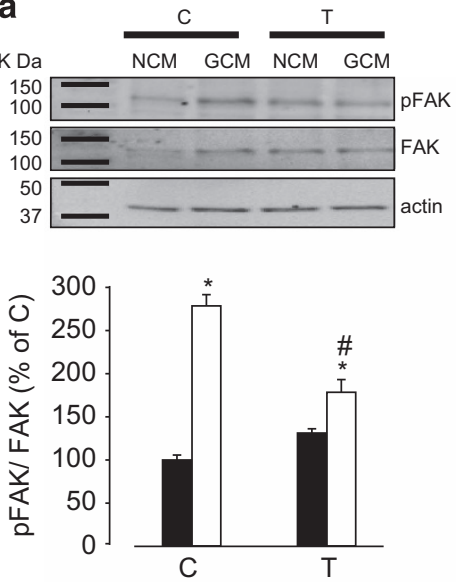
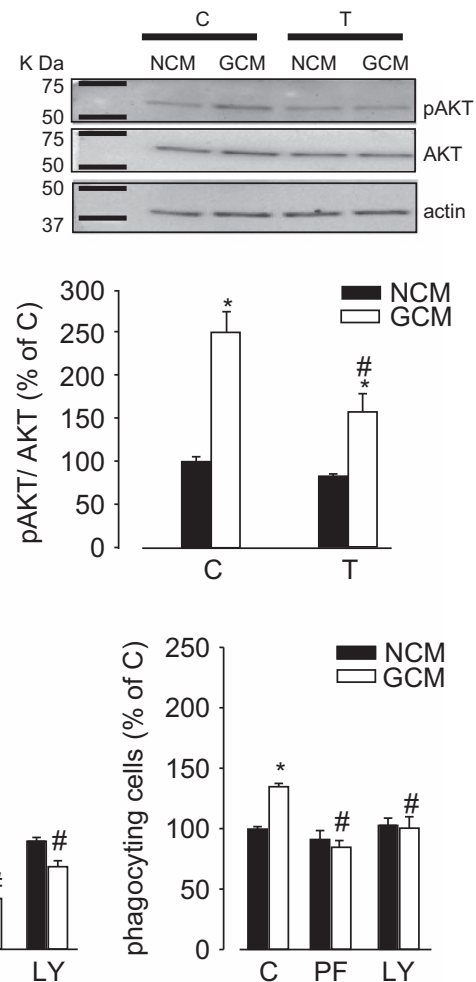

C
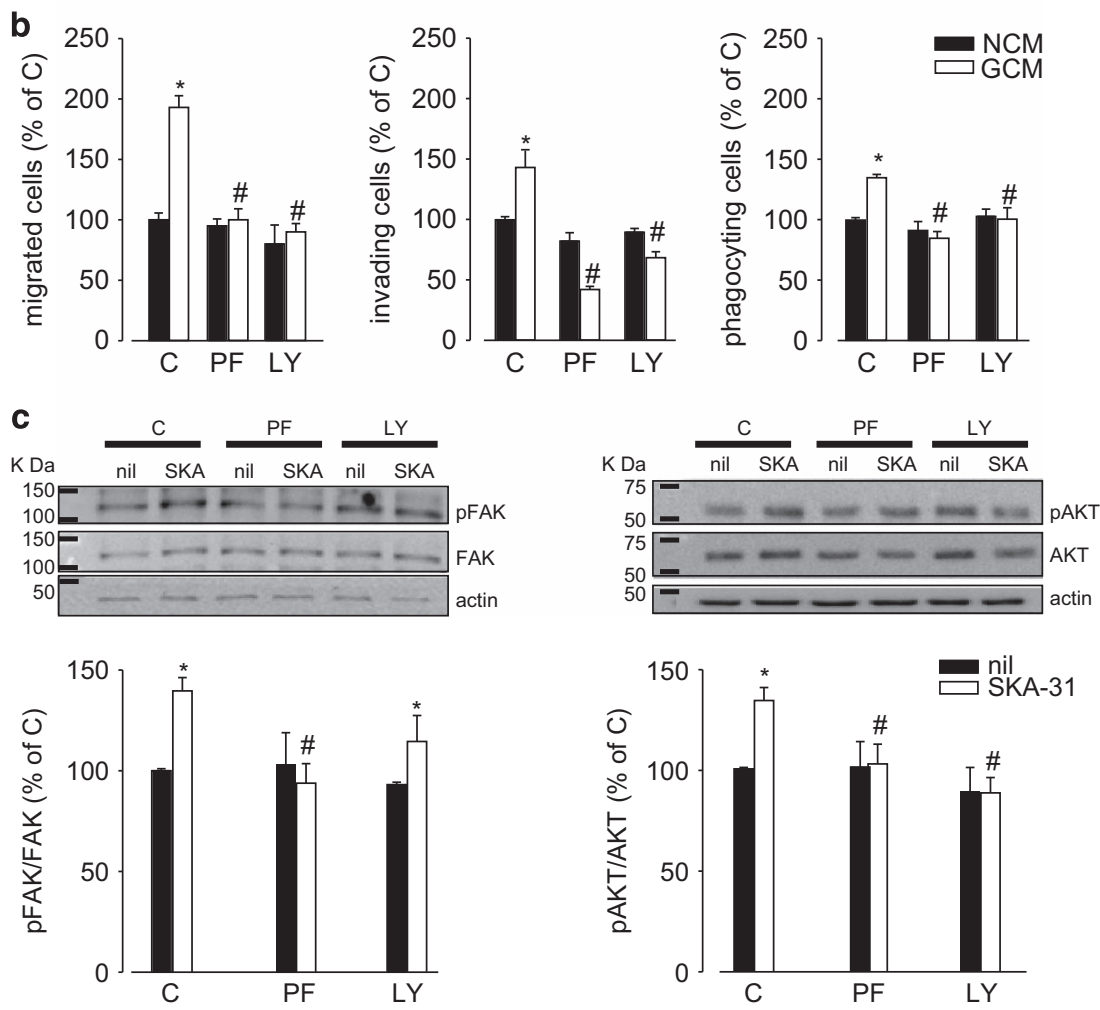

Figure 5 Signaling pathways involved in KCa3.1 channel activation. (a) Levels of pFAK/FAK and pAKT/AKT in NCM- and GCM-treated microglia in the absence (C) or presence of TRAM-34 (T, $2.5 \mu \mathrm{M})$. Data are the mean \pm S.E.M., ${ }^{*} P<0.05$ versus $\mathrm{NCM} ;{ }^{\#} P<0.05$ versus $\mathrm{GCM} / \mathrm{C} ; N=5$. Representative blots are shown on the top. Actin was used as a loading control, and molecular markers are indicated on the left (KDa). (b) Migration, invasion and phagocytosis assays on NCM- and GCM-treated microglia in the absence $(\mathrm{C})$ or presence of PF-228 (PF, $10 \mu \mathrm{M})$ or LY294002 (LY, $25 \mu \mathrm{M})$. Data are the mean \pm S.E.M., ${ }^{*} P<0,05$ versus NCM; ${ }^{*} P<0.05$ versus GCM/C; $N=4$. (c) Levels of pFAK/FAK and pAKT/AKT in untreated or SKA-31-treated (250 nM) microglia in the absence (C) or presence of PF-228 (PF, $10 \mu \mathrm{M})$ or LY294002 (LY, $25 \mu \mathrm{M})$. Data are shown as mean \pm S.E.M.; ; $P<0.05$ versus $N C M$; ${ }^{~} P<0.05$ versus $\mathrm{GCM} / \mathrm{C} ; N=8$. Top: representative blots are shown on the top. Actin was used as a loading control, and molecular markers are indicated on the left $(\mathrm{KDa})$

macrophage toward a pro-inflammatory phenotype was also described for another cation channel, TRPM7, ${ }^{27}$ and a recent reports describe that CSFR-1 inhibition has similar effects on the polarization of tumor-associated macrophages (TAM), downregulating M2-related genes, with consequent antitumor effects. $^{28}$ In accordance, inhibition of the mTOR kinase has been reported to induce the polarization of glioma-induced microglia toward the pro-inflammatory state. ${ }^{29}$ It is known that glioma-exposed microglia have an increased tendency to migrate, invade and phagocyte ${ }^{14}$ here we also observed that KCa3.1 inhibition affected these microglia functions, likely contributing to the antitumor activity of TRAM-34, in addition to the direct effect on glioma. ${ }^{14}$ We also reported that microglia treated with the KCa3.1 channel activator SKA-31, or polarized by IL-4, had similar increased migratory, invasive and phagocytic activities, all significantly reduced by KCa3.1 
a
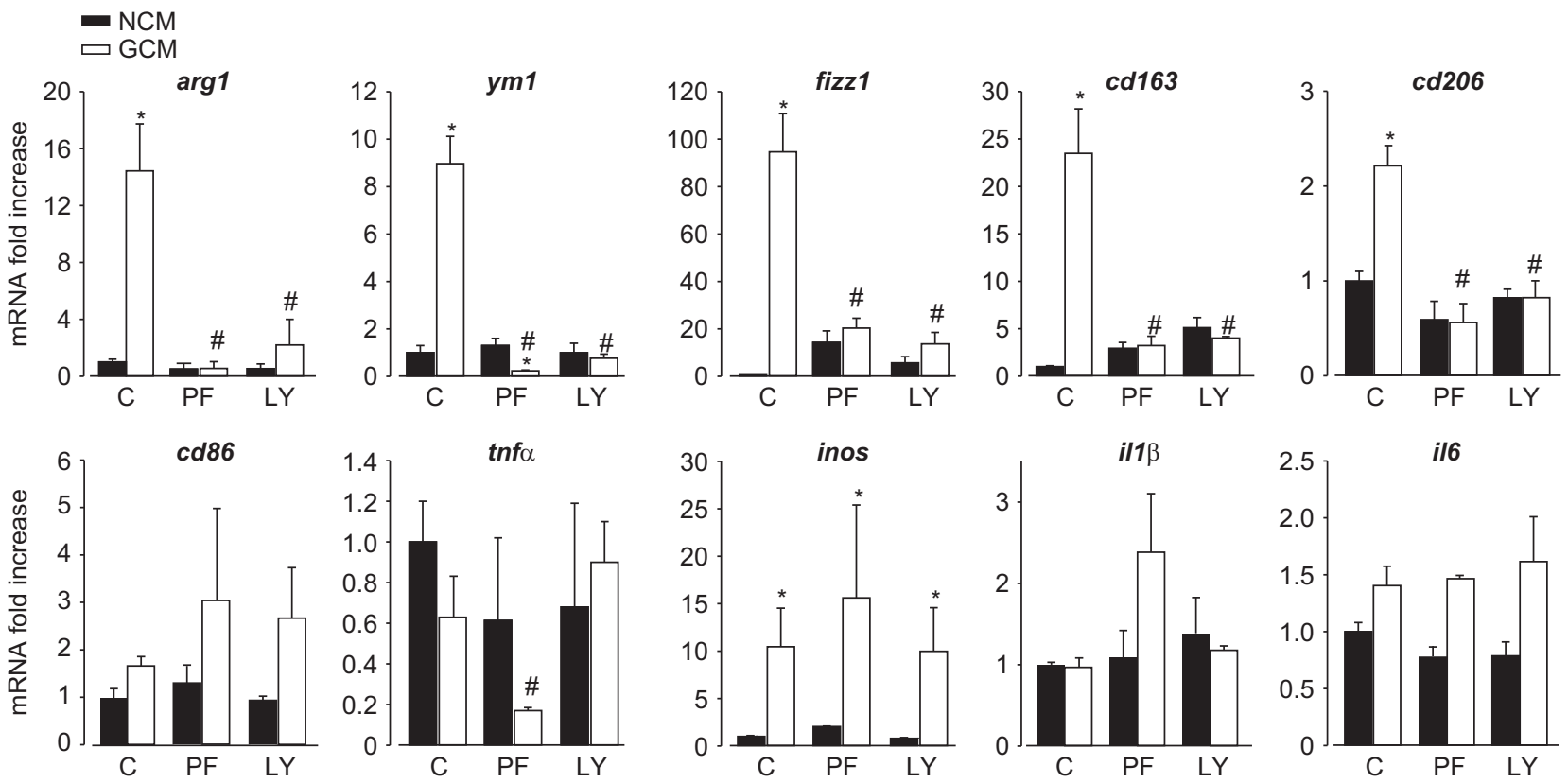

b
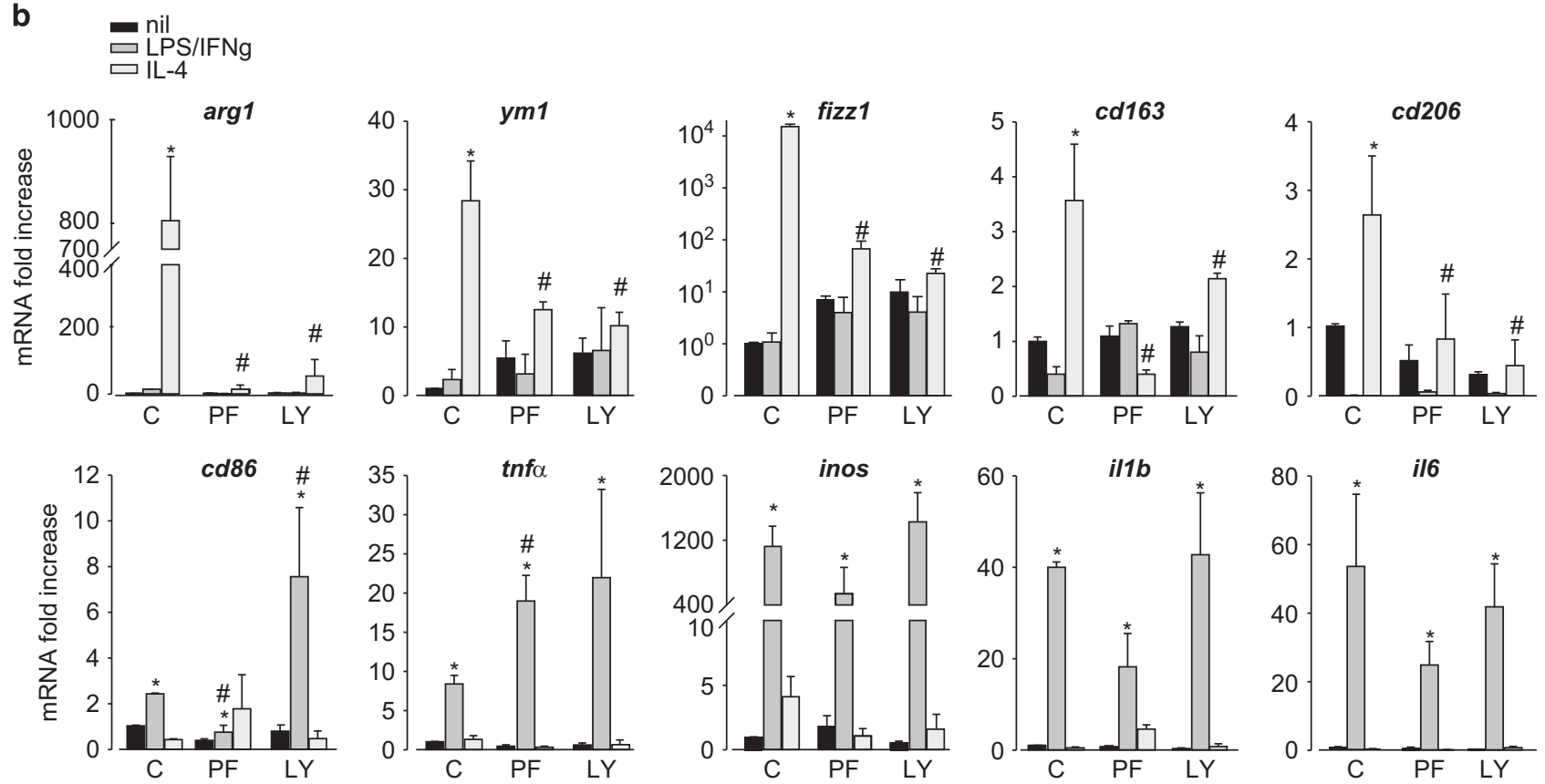

Figure 6 FAK and AKT kinases are required for the anti-inflammatory phenotype of microglia. (a) RT-PCR on mRNAs of microglia treated with NCM or GCM in the absence (C) or presence of PF-228 (PF, $10 \mu \mathrm{M})$ or LY294002 (LY, $25 \mu \mathrm{M})$, assayed for the expression of anti- (arg1, ym1, fizz1, cd163 and cd206) and pro- (cd86, tnfo, inos, il1 $\beta$ and il6) inflammatory genes. Data are expressed as fold increase and are the mean \pm S.E.M.; ${ }^{*} P<0.05$ versus $N C M$; $\# P<0.05$ versus $G C M / C$ by two-way ANOVA; $N=8$. (b) RT-PCR on mRNAs of untreated, LPS/IFN $\gamma$ - or IL-4-treated microglia in the absence (C) or presence of PF-228 (PF, $10 \mu \mathrm{M})$ or LY294002 (LY, $25 \mu \mathrm{M})$, assayed for the expression of anti(arg1, ym1, fizz1, $c d 163$ and $c d 206)$ and pro- (cd86, tnfo, inos, il1 $\beta$ and il6) inflammatory genes. Data are expressed as fold increase and are the mean \pm S.E.M.; ${ }^{*} P<0.05$ versus nil; ${ }^{\#} P<0.05$ versus LPS/IFN $\gamma / C$ or versus IL4/C by two-way ANOVA; $N=4$

inhibition. Microglia migration varies according to their activation state. ${ }^{30,31}$ We report that IL-4-treated microglia upregulated the chemokine receptors CXCR4 and CXCR6 and the MMP-9, all effects being abolished by TRAM-34. Interestingly, LPS/IFN $\gamma$ treatment did not modify CXCR6 or MMP-9 expression, strongly inhibiting CXCR4 expression. This reduction could contribute to the antitumor effect reported for microglia in the pro-inflammatory state. ${ }^{12} \mathrm{IL}-4$ treatment consistently increased microglia migration toward CXCL12 and CXCL16. Interestingly, similar reduction of MMP-12 is induced by TRAM-34 treatment of CD11b+ cells isolated from GBM patients. These results confirm that the 
anti-inflammatory phenotype of microglia can be reverted by TRAM-34, which, however, also affects some classic proinflammatory features of microglia. ${ }^{16,18,19}$

Remarkably, we report that TRAM-34 did not affect microglia functions (migration, invasion, phagocytosis) in the absence of stimulation or upon LPS/IFN $\gamma$ treatment, in contrast to the inhibitory effects observed on activating pathways induced by inflammatory stimuli, such as oligomeric amyloid and LPS. ${ }^{19}$ This could be explained by the lower expression level of kcnn4 and the lower functional expression of KCa3.1 in comparison with anti-inflammatory phenotype, in our experimental systems, that comprise in vivo, ex vivo and in vitro conditions.

To describe the intracellular pathways involved in KCa3.1mediated control of microglia phenotype, we analyzed FAK and PI3K/AKT signaling that have been demonstrated to be activated in TAMs and involved in the M2-like microglia polarization. ${ }^{23}$ We demonstrated that both kinases are essential in movement, phagocytosis and invasion of GCMand IL-4-treated cells; that FAK and PI3K/AKT signaling in microglia is downstream of $\mathrm{KCa} 3.1$ activation and that the block of FAK phosphorylation hampers SKA-31-induced PI3K/AKT activation. We hypothesize that KCa3.1 activation could induce local $\mathrm{Ca}^{2+}$ influx leading to FAK and AKT phosphorylation $^{32,33}$ and that these signals are involved in GCM- and IL-4-mediated microglia activation and polarization. KCa3.1 inhibition with TRAM-34 inhibited all these events, promoting the induction of antitumor signals.

Our data suggest that, in addition to being involved in modulating microglia phenotype, KCa3.1 channels are differentially expressed in the different activation states of microglia. Microglia isolated from the brain of glioma-bearing mice and from biopsies of patients with glioma had significant upregulation of kcnn4, as well as microglia exposed to GCM or treated with IL-4 (see also Ferreira et al. $^{34}$ ). In all these samples, KCa3.1 inhibition reduced kcnn4 expression, as already described in human lung myofibroblasts and vascular smooth muscle cells, ${ }^{35-37}$ suggesting a clear relation between microglia activation state and the control of KCa3.1 transcription. A relation between $\mathrm{KCa} 3.1$ expression level and activation was already reported in $\mathrm{T}$ cells, where a $\mathrm{Ca}^{2+}$ dependent mechanism involves JNK and AP1 (c-Fos/c-Jun heterodimer) complex binding to the kcnn4 promoter. ${ }^{38}$

In summary, based on the data herein presented, we propose $\mathrm{KCa} 3.1$ as a possible marker of $\mathrm{M} / \mathrm{M} \Phi$ in the protumor state, and thus a potential therapeutic target to induce GBM-associated $\mathrm{M} / \mathrm{M} \Phi$ to re-acquire a pro-inflammatory, antitumor activity. Of note, and important for future therapeutic use, one TRAM-34 analog, Senicapoc (ICA-17043), has been already used in phase-3 clinical trials, where it was considered safe, with no clinically relevant changes in vital signs and physical examination. ${ }^{39}$

\section{Materials and Methods}

Animals and cell lines. Experiments were approved by the Italian Ministry of Health in accordance with the ethical guidelines on use of animals from the EC Council Directive 2010/63/EU. We used C57BL/6 mice (Charles River, Calco, Italy). Human GBM (GL-15 and U87MG) and murine glioma (GL261) cell lines were cultured in FBS-supplemented DMEM.
Microglia polarization. Microglia were obtained from cerebral cortices of postnatal day 0-2 mice ${ }^{40}$ and treated for $24-48 \mathrm{~h}$ with LPS $100 \mathrm{ng} / \mathrm{ml}+\mathrm{IFN} \gamma$ $20 \mathrm{ng} / \mathrm{ml}$ (ImmunoTools, Friesoythe, Germany) or IL-4 $20 \mathrm{ng} / \mathrm{ml}$ (ImmunoTools) for cell polarization.

Patch clamp. Patch-clamp recordings were obtained using glass electrodes (2-4 M 2 ) filled with intracellular solution (see composition in Supplementary Methods). Currents were recorded by whole-cell configuration at RT with EPC-10HEKA amplifier (HEKA, Lambrecht/Pfalz, Germany). $\mathrm{K}^{+}$currents were elicited with voltage ramps $(-120 \mathrm{mV}$ to $+40 \mathrm{mV}, 200 \mathrm{~ms})$ every $10 \mathrm{~s}$. KCa3.1 conductances were calculated from the slope of the TRAM-34-sensitive KCa current between -80 and $-75 \mathrm{mV}$, where $\mathrm{KCa} 3.1$ currents are not 'contaminated' by Kv1.3 (activable at voltages $>-40 \mathrm{mV}$ ) or inward rectifier $\mathrm{K}^{+}$currents (appreciable at voltages $<-80 \mathrm{mV}$ ). Cell capacitance was continuously monitored. $\mathrm{KCa} 3.1$ current density was determined as the TRAM-34-sensitive slope conductance/cell capacitance. The prominent Kv current in pro-inflammatory microglia was blocked with Kv1.3 blocker PAP-1 $(1 \mu \mathrm{M})$.

Chemotaxis and invasion assays. Microglia stimulated with NCM or GCM, and cytokine-polarized microglia were treated for $24 \mathrm{~h}$ with TRAM-34 $(2.5 \mu \mathrm{M})$ or without. Cells were incubated $3 \mathrm{~h}$ at $37^{\circ} \mathrm{C}$ with $10 \% \mathrm{FBS}$ in the lower chamber as chemoattractant or with chemokines (CXCL12, $50 \mathrm{nM}$; CXCL16, $1 \mathrm{nM}$, Peprotech, London, UK). For invasion assay, microglia were plated on matrigelcoated transwells (BD-Falcon, Milan, Italy) and the movement toward GCM (from GL261, GL15 or U87MG) was investigated in the presence of TRAM-34 $(2.5 \mu \mathrm{M})$ for $24 \mathrm{~h}$.

Phagocytosis. Microglial cells were stimulated with GCM for $24 \mathrm{~h}$ with TRAM-34 $(2.5 \mu \mathrm{M})$. Alternatively, polarized microglia were treated with TRAM-34 $(2.5 \mu \mathrm{M})$ for $24 \mathrm{~h}$. Red fluorescent FluoSpheres $(0.03 \%)$ (Invitrogen, Monza, Italy) were added for $1 \mathrm{~h}$, and the number of spheres per cell was counted. ${ }^{14}$

Real-time PCR. GCM-stimulated or cytokine-polarized microglia were treated with TRAM-34 $(2.5 \mu \mathrm{M})$. After $24 \mathrm{~h}$, total RNA was extracted with Trizol reagent (Invitrogen), quantified and retro-transcripted using IScript Reverse Transcription Supermix (Bio-Rad, Milan, Italy). RT-PCR was carried out in a I-Cycler IQ Multicolor RT-PCR Detection System (Bio-Rad) using SsoFast Eva Green Supermix (Bio-Rad). The PCR protocol consisted of 40 cycles of denaturation at $95^{\circ} \mathrm{C}$ for $30 \mathrm{~s}$ and annealing/extension at $58^{\circ} \mathrm{C}$ for $30 \mathrm{~s}$. The $\mathrm{Ct}$ values from each gene were normalized to the $\mathrm{Ct}$ value of GAPDH. Relative quantification was performed using the $2^{-\Delta \Delta C t}$ method and expressed as fold increase. Primer sequences are in Supplementary Table S2.

Form factor calculation. Microglia were seeded on glass coverslips, treated as necessary, fixed, permeabilized, blocked and stained with Alexa-Fluor 488 Phalloidin (Invitrogen) for 20 min together with Hoechst. Fluorescent images were processed using the MetaMorph 7.6.5.0 software (Molecular Device, Sunnyvale, CA, USA), and form factor was calculated according the formula: $4 \pi$ area/ perimeter ${ }^{2}{ }^{41}$

Western blotting analysis. For protein phosphorylation analysis, protein samples were separated on $8.75 \%$ SDS-polyacrylamide gel electrophoresis and analyzed by western immunoblot using the following primary antibodies: pFAK (Tyr397, Santa Cruz Biotechnology, Santa Cruz, CA, USA) 1: 200, pAKT (Ser473, Cell Signaling, Danvers, MA, USA) 1:1000, FAK (Santa Cruz Biotechnology) $1: 200$, AKT (Cell Signaling) $1: 1000$, and Actin (Sigma Aldrich, Milan, Italy) $1: 2000$; HRP-tagged goat anti-rabbit IgG was used as a secondary antibody ( 1 : 2000; Dako, Cernusco sul Naviglio, Milan, Italy); the detection was performed through the chemiluminescence assay Immun-Star Western C Kit (Bio-Rad). Densitometric analysis was carried out with the Quantity One software (Bio-Rad).

Tumor cell implantation and mice treatment. Eight-week-old male C57BL/6 mice were anesthetized with chloralhydrate $(400 \mathrm{mg} / \mathrm{kg}$, i.p.) and stereotaxically injected with $1 \times 10^{5}$ GL261 cells in $5 \mu$ PBS, $2 \mathrm{~mm}$ right and $1 \mathrm{~mm}$ anterior to the bregma in the striatum at $3 \mathrm{~mm}$ depth with a Hamylton syringe (Bonaduz, Switzerland). After 7 days, mice were daily treated with TRAM-34 $(120 \mathrm{mg} / \mathrm{Kg}$ i.p.) or vehicle (peanut oil). 
Tumor volume analysis. After 17 days from GL261 injection, animals were killed and the brains were isolated. Tumor volume was evaluated with hematoxylineosin staining as previously described. ${ }^{42}$ Briefly, after staining, brain slices $(20 \mu \mathrm{m}$ of thickness) were analyzed by the Image Tool 3.0 software (University of Texas, Health Science Center, San Antonio, TX, USA). to measure the tumor area, and volume was calculated according to the formula (volume $=t \times \Sigma A$ ), where $A=$ tumor area/slice and $t=$ thickness. $^{43,44}$

Isolation of $\mathbf{C D}_{11 b^{+}}$cells. After 17 days from GL261 injection, mice were deeply anesthetized and perfused with ice-cold PBS. Brains were removed and digested with trypsin $(0.25 \mathrm{mg} / \mathrm{ml})$. Tissue suspension was applied to a $30-\mu \mathrm{m}$ cell strainer, labeled with CD11b MicroBeads and isolated accordingly to the manufacturer's instructions (Miltenyi Biotec, Calderara di Reno, Bologna, Italy). $\mathrm{CD} 11 \mathrm{~b}+$ cell purity $(99 \%)$ was verified as reported. ${ }^{42}$

Immunofluorescence. Coronal brain sections $(20 \mu \mathrm{m})$ were washed in PBS, blocked ( $3 \%$ goat serum in $0.3 \%$ Triton $\mathrm{X}-100$ ) for $1 \mathrm{~h}$ at RT and incubated overnight at $4{ }^{\circ} \mathrm{C}$ with specific antibodies, Iba1 (Dako) 1:500 and KCa3.1 (Alomone, Jerusalem, Israel) $1: 100$. Brain slices were stained with the fluorophore-conjugated secondary antibodies and Hoechst for nuclei visualization and analyzed using a fluorescence microscope. Signals co-localization was analyzed measuring the average fluorescence intensity (pixel) of merged signals.

Isolation of $\mathrm{CD} 1 \mathrm{~b}^{+}$cells from human GBM. Tumor specimens obtained from adult patients who gave informed consent to the research proposals (Neurosurgery Departments, Neuromed and Policlinico Umberto I) were processed as described above to isolate $\mathrm{CD}_{11 \mathrm{~b}^{+}}$cells. See Supplementary Table S1 for patients' details. Cells were treated with TRAM-34 $(2.5 \mu \mathrm{M})$ for $24 \mathrm{~h}$ and mRNAs were analyzed by RT-PCR for human pro- and anti-inflammatory markers (primer sequences are listed in Supplementary Table S2).

Statistical analysis. Data are expressed as the means \pm S.E.M. Student's $t$-test, paired $t$-test, one-way or two-way analysis of variance (ANOVA) was performed. A value of $P<0.05$ was considered significant. All statistical analyses were carried out using the Sigma Plot 11.0 Software (Systat Software GmbH, Erkrath, Germany).

\section{Conflict of Interest}

The authors declare no conflict of interest.

Acknowledgements. We thank Dr. Flavia Trettel and Dr. Clotilde Lauro for helpful discussions, Dr. Serena Pellegatta for GL261 cells and Dr. Giuseppina Chece and Dr. Andrea Mele for help with microsurgery. This work was supported by AIRC IG2012-12774 to CL and by a National Institute of General Medicine Award GM076063 to HW.

1. Stupp R, Mason WP, Van Den Bent MJ, Weller M, Fisher B, Taphoorn MJB et al. Radiotherapy plus con-comitant and adjuvant temozolomide for glioblastoma. $N$ Engl J Med 2005; 352: 987-996.

2. Charles NA, Holland EC, Gilbertson R, Glass R, Kettenmann H. The brain tumor microenvironment. Glia 2012; 60: 502-514.

3. Graeber MB, Scheithauer BW, Kreutzberg GW. Microglia in brain tumors. Glia 2002; 40: 252-259.

4. Nimmerjahn A, Kirchhoff F, Helmchen F. Resting microglial cells are highly dynamic surveillants of brain parenchyma in vivo. Science 2005; 308: 1314-1318.

5. Hanisch UK, Kettenmann H. Microglia: active sensor and versatile effector cells in the normal and pathologic brain. Nat Neurosci 2007; 10: 1387-1394.

6. Kettenmann H, Hanisch UK, Noda M, Verkhratsky A. Physiology of microglia. Physiol Rev 2011; 91: 461-553.

7. Benoit M, Desnues B, Mege JL. Macrophage polarization in bacterial infections. $J$ Immunol 2008; 181: 3733-3739.

8. Martinez FO, Sica A, Mantovani A, Locati M. Macrophage activation and polarization. Front Biosci 2008; 13: 453-461.

9. Komohara $Y$, Ohnishi K, Kuratsu J, Takeya M. Possible involvement of the M2 anti inflammatory macrophage phenotype in growth of human gliomas. J Pathol 2008; 216: $15-24$.

10. Li W, Graeber MB. The molecular profile of microglia under the influence of glioma. NeuroOncol 2012; 14: 958-978.
11. Pollard JW. Tumour-educated macrophages promote tumour progression and metastasis. Nat Rev Cancer 2004; 4: 71-78.

12. Gabrusiewicz K, Ellert-Miklaszewska A, Lipko M, Sielska M, Frankowska M, Kaminska B. Characteristics of the alternative phenotype of microglia/macrophages and its modulation in experimental gliomas. PLOS One 2011; 6: e23902.

13. Sica A, Mantovani A. Macrophage plasticity and polarization: in vivo veritas. J Clin Invest 2012; 122: 787-795.

14. D'Alessandro G, Catalano M, Sciaccaluga M, Chece G, Cipriani R, Rosito M et al. KCa3.1 channels are involved in the infiltrative behavior of glioblastoma in vivo. Cell Death Dis 2013; 4: e773.

15. Bouhy D, Ghasemlou N, Lively S, Bouhy D, Ghasemlou N, Lively S et al. Inhibition of the $\mathrm{Ca} 2+-$ dependent $\mathrm{K}+$ channel, $\mathrm{KCNN} 4 / \mathrm{KCa} 3.1$, improves tissue protection and locomotor recovery after spinal cord injury. J Neurosci 2011; 31: 16298-16308.

16. Chen YJ, Raman G, Bodendiek S, O'Donnell ME, Wulff H. The KCa3.1 blocker TRAM-34 reduces infarction and neurological deficit in a rat model of ischemia/reperfusion stroke. J Cereb Blood Flow Metab 2011; 31: 2363-2374.

17. Maezawa I, Jenkins DP, Jin BE, Wulff H. Microglial KCa3.1 channels as a potential therapeutic target for Alzheimer's disease. Int J Alzheimers Dis 2012; 2012: 868972.

18. Kaushal V, Koeberla PD, Wang Y, Schlichter LC. The Ca2+- activated K+ channel KCNN4/ $\mathrm{KCa} 3.1$ contributes to microglia activation and nitric oxide-dependent neurodegeneration. J Neurosci 2007; 27: 234-244.

19. Maezawa I, Zimin PI, Wulff H, Jin LW. Amyloid-beta protein oligomer at low nanomolar concentrations activates microglia and induces microglial neurotoxicity. J Biol Chem 2011; 286: 3693-3706.

20. Turner KL, Honasoge A, Robert SM, McFerrin MM, Sontheimer H. A proinvasive role for the $\mathrm{Ca}^{(2+)}$-activated $\mathrm{K}^{(+)}$channel KCa3.1 in malignant glioma. Glia 2014; 62: 971-981.

21. Murray PJ, Allen JE, Biswas SK, Fisher EA, Gilroy DW, Goerdt S et al. Macrophage activation and polarization: nomenclature and experimental guidelines. Immunity 2014; 41 : $14-20$.

22. Ellert-Miklaszewska A, Dabrowski M, Lipko M, Sliwa M, Maleszewska M, Kaminska B. Molecular definition of the pro-tumorigenic phenotype of glioma-activated microglia. Glia 2013; 61: 1178-1190.

23. Tarassishin L, Suh HS, Lee SC. Interferon regulatory factor 3 plays an anti-inflammatory role in microglia by activating the PI3K/Akt pathway. J Neuroinflammation 2011; 8: 187.

24. Hanks SK, Ryzhova L, Shin NY, Brabek J. Focal adhesion kinase signaling activities and their implications in the control of cell survival and motility. Front Biosci 2003; 8: d982-d996.

25. Sun X, Wang X, Chen T, Li T, Cao K, Lu A et al. Myelin activates FAK/Akt/NF-kappaB pathways and provokes CR3-dependent inflammatory response in murine system. PLoS One 2010; 5: e9380.

26. Badie B, Schartner J. Role of microglia in glioma biology. Microsc Res Tech 2001; 54: 106-113.

27. Schilling T, Miralles F, Eder C. TRPM7 regulates proliferation and polarisation of macrophages. J Cell Sci 2014; 127: 4561-4566.

28. Pyonteck SM, Akkari L, Schuhmacher AJ, Bowman RL, Sevenich L, Quail DF et al. CSF-1R inhibition alters macrophage polarization and blocks glioma progression. Nat Med 2013; 19: 1264-1272.

29. Lisi L, Laudati E, Navarra P, Dello Russo C. The mTOR kinase inhibitors polarize glioma-activated microglia to express a M1 phenotype. J Neuroinflammation 2014; 11: 125.

30. Lively S, Schlichter LC. The microglial activation state regulates migration and roles of matrix-dissolving enzymes for invasion. J Neuroinflammation 2013; 10: 75.

31. Siddiqui T, Lively S, Ferreira R, Wong R, Schlichter LC. Expression and contributions of TRPM7 and KCa2.3/SK3 channels to the increased migration and invasion of microglia in anti-inflammatory activation states. PLOS One 2014; 9: e106087.

32. Ferreira R, Schlichter LC. Selective activation of KCa3.1 and CRAC channels by P2Y2 receptors promotes $\mathrm{Ca}(2+)$ signaling, store refilling and migration of rat microglial cells. PLOS One 2013; 8: e62345.

33. Giannone G, Rondé P, Gaire M, Haiech J, Takeda K. Calcium oscillations trigger focal adhesion disassembly in human U87 astrocytoma cells. J Biol Chem 2002; 277: 26364-26371.

34. Ferreira R, Lively S, Schlichter LC. IL-4 type 1 receptor signaling up-regulates KCNN4 expression, and increases the $\mathrm{KCa} 3.1$ current and its contribution to migration of alternativeactivated microglia. Front Cell Neurosci 2014; 8: 183.

35. Roach KM, Feghali-Bostwick C, Wulff H, Amrani Y, Bradding P. Human lung myofibroblast TGF 31 -dependent Smad2/3 signalling is $\mathrm{Ca}(2+)$-dependent and regulated by $\mathrm{KCa} 3.1 \mathrm{~K}(+)$ channels. Fibrogenesis Tissue Repair 2015; 8: 5.

36. Tharp DL, Wamhoff BR, Wulff H, Raman G, Cheong A, Bowles DK. Local delivery of the KCa3.1 blocker, TRAM-34, prevents acute angioplasty-induced coronary smooth muscle phenotypic modulation and limits stenosis. Arterioscler Thromb Vasc Biol 2008; 28 : 1084-1089.

37. Cheong A, Bingham AJ, Li J, Kumar B, Sukumar P, Munsch C et al. Downregulated REST transcription factor is a switch enabling critical potassium channel expression and cell proliferation. Mol Cell 2005; 20: 45-52.

38. Ghanshani S, Wulff H, Miller MJ, Rohm H, Neben A, Gutman GA et al. Up-regulation of the IKCa1 potassium channel during T-cell activation. Molecular mechanism and functional consequences. J Biol Chem 2000; 275: 37137-37149. 
39. Ataga KI, Stocker J. Senicapoc (ICA-17043): a potential therapy for the prevention and treatment of hemolysis-associated complications in sickle cell anemia. Expert Opin Investig Drugs 2009; 18: 231-239.

40. Lauro C, Cipriani R, Catalano M, Trettel F, Chece G, Brusadin V et al. Adenosine A1 receptors and microglial cells mediate $\mathrm{CX} 3 \mathrm{CL} 1$-induced protection of hippocampal neurons against Glu-induced death. Neuropsychopharmacol 2010; 35: 1550-1559.

41. Neubrand VE, Pedreño M, Caro M, Forte-Lago I, Delgado M, Gonzalez-Rey E. Mesenchymal stem cells induce the ramification of microglia via the small RhoGTPases Cdc42 and Rac1. Glia 2014; 62: 1932-1942.

42. Garofalo S, D'Alessandro G, Chece G, Brau F, Maggi L, Rosa A et al. Enriched environment reduces glioma growth through immune and non-immune mechanisms in mice. Nat Commun 2015; 6: 6623.

43. Mayhew TM, Mwamengele GL, Dantzer V. Stereological and allometric studies on mammalian cerebral cortex with implications for medical brain imaging. J Anat 1996; 189(Pt 1): 177-184.

\section{Roberts N, Puddephat MJ, McNulty V. The benefit of stereology for quantitative radiology. Br J Radiol 2000; 73: 679-697.}

(c) (i) Cell Death and Disease is an open-access journal published by Nature Publishing Group. This work is licensed under a Creative Commons Attribution 4.0 International License. The images or other third party material in this article are included in the article's Creative Commons license, unless indicated otherwise in the credit line; if the material is not included under the Creative Commons license, users will need to obtain permission from the license holder to reproduce the material. To view a copy of this license, visit http://creativecommons.org/licenses/by/4.0/

Supplementary Information accompanies this paper on Cell Death and Disease website (http://www.nature.com/cddis) 